\title{
Type IV Hypersensitivity
}

National Cancer Institute

\section{Source}

National Cancer Institute. Type IV Hypersensitivity. NCI Thesaurus. Code C3115.

Delayed hypersensitivity reaction (DTH) - type IV reaction, an inflammatory response that develops 24 to 72 hours after exposure to an antigen that the immune system recognizes as foreign. DTH is mediated by T cells rather than by antibodies. Th1 cells produce interferon gamma, interleukin (IL)-2, and tumor necrosis factor-beta and promote a cell-mediated immune response. 